The Dominant Mode of Standing Alfvén Waves at the

Synchronous Orbit

by

W.D. Cummings, Calvin Countee, Donald Lyons and William Wiley, III

Department of Physics

Grambling State University

Gramb ling, LA 71245

January 18, 1975 


\author{
The Dominant Mode of Standing Alfvén Waves \\ at the \\ Svnchronous Orbit \\ by \\ W.D. Cummings, Calvin Countee, Donald Lyons \\ and William Wiley, III \\ Department of Physics \\ Grambling State University \\ Grambling, LA 71245
}

\begin{abstract}
Low-frequency oscillations of the earth's magnetic field recorded bv the UCLA magnetometer on board ATS-1, have been examined for the six-month interval, January-June, 1968. The initial interpretation, (Cumings et al., 1969) that these oscillations represent the second harmonic of a standing Alfvén wave, has been re-examined, and it is concluded that this hypothesis must be withdrawn. Using evidence from $0 G O-5$ and ATS-5, as well as the data from ATS-1, we argue that the dominant mode at the synchronous orbit must be the fundamental rather than the second harmonic. From 14 instances when the oscillations of distinctly different periods occurred during the same time interval at ATS-1 we also conclude that higher hamonics can exist. The period ratio in 7 of the 14 cases corresponds to the simultaneous occurrence of the second harmonic with the fundamental, and 4 other cases could be identified as the simultaneous occurrence of the fourth hamonic with the fundamental.
\end{abstract}




\author{
The Dominant Mode of Standing Alfvén Waves \\ at the \\ Synchronous orbit \\ by \\ W.D. Cummings, Calvin Countee, Donald Lyons \\ and William Willey, III \\ Department of Physics \\ Grambling State University \\ Grambling, LA 71245
}

Lanzerotti and Fukuniski (1974) have recently given a review of experimental and theoretical work related to the modes of magnetospheric Alfvern waves. The goal of their review was to raise the question of whether the dominant mode was odd or even. They conclude from their own conjugate data that near $L=4$ the dominant mode is odd.

Lanzerotti and Eukuniski correctly point out that in our initial paper (Cumings et al., 1969) we assumed that the oscillations observed at ATS-1 were even mode, primarily because ATS-1 is located on the magnetic equator, and at least in our idealized model, this position represents a node for standing, Alfvén waves of odd mode. It should also be noted that our initial analysis was based on only 25 events during January, 1968 and that we only studied oscillations in the period range 50-300 seconds. This latter limitation of the period range resulted from the fact that we analyzed 15 second averages of the data. Therefore, our ability to observe oscillations dimished with decreasing period, and was certainly marginal near a period of 50 seconds.

This limitation tumed out to be critical, as it is now apparent from the present analysis that a dominant period at the synchronous orbit is in the range $40-90$ seconds. 
In a second analysis (Cummings et al., 1972) we analyzed 222 events over a 25-month data interval. However, 15 second averages of the data were also used in this study, so the limitation at low periods still existed.

The present analysis is based on 273 events that were recorded in the sixmonth interval, January-June, 1968. The minimum period recorded was 20.7 secs. and the maximum period was 410.8 seconds. An artificial oscillation at a period of 5.12 seconds, the interval of one data sequence, sometimes contaminated the data. Therefore, a practical lower limit to the periods we could observe is approximately 20 seconds, even though we used 0.32 second averages of the data in the analysis.

ATS- 1 was located at $150^{\circ} \mathrm{H}$ longitude, which is approximatelv the intersection of the geomagnetic and geographic equatorial planes. The magnetometer experiment on board ATS-l has been described in Cummings et al. (1969) and in Cummings and Coleman (1968). It should be mentioned that the plasma detector scheduled for ATS-1 failed prior to launch, and the remaining particle detectors did not provide a complete spectrum of the ambient charged particle population. It has, therefore, been impossible to determine the ambient total ion number density from ATS-1 particle data. In this study, we have made use of OGO-5 data and published ATS-5 data to help fill this unfortunate gap in the ATS-1 data.

\section{GENERAL RESULTS}

of the 273 events that occurred during the six-month interval, 25 were recognized as being similar to those described by Barfield et al., (1972). That is, they were compressional, usually related to storms on substorms, and occurred in the dusk sector. We have excluded these events from this analysis. 
The distribution of periods for the remaining 248 events is shown as a histogram in Figure 1. The most striking feature of the histogram is the peak in the range $40-90$ seconds. The most frequently occurring period is in the range 60-70 seconds. We cannot rule out an additional peak at periods below 20 seconds, which are unobservable in the ATS-I data, as explained above. The average period for the 248 events was $T_{\text {ave }}=77.4$ seconds.

The distribution of the 248 events in local time is shown in Figure 2 . In the histogram of Figure 2, the ordinate represents the number of hours in which oscillations occurred in a given local time interval. The peak of the local time distribution is $1-3$ hours past noon, but the distribution is asymmetric, favoring the morning hours.

This distribution is broadly similar to that observed at 6.25 earth radii with the DODGE experiment (Dwarkin et a1., 1971) in that both distributions show that the great majority of oscillations occur in the day sector. The local time distribution in our second analysis (Cummings et al., 1972; Figure 2) was similar in shape to the present distribution with the peak also shifted slightly toward the afternoon sector. We note again that the period range in the second analysis was limited to $T>50$ seconds because 15 second averages of the data were used.

In our first analysis (Cummings et al., 1969) we reported that the oscillations were observed predominately on geomagnetically quiet days. It is cerm tainly true that many of the longer period oscillations are found to occur during the quiet recovery phase of magnetic storms. However, it is clear from the present analysis that one should not associate the more typical oscillations observed at the synchronous orbit with prolonged periods when the magnetosphere is quiet. On the contrary, these oscillations seem to be more clearly related to magnetic storms or periods of magnetic activity. 
To illustrate the above point, we used the hourly values of equatorial Dst (Sugiura and Poros, 1971) for January-June, 1968, to identify the hours when the Dst value was at a local minimum. We required the local minimum to be greater, in absolute value, than $35 \%$ and 50 such instances were found. Next, we compared this set of times, $t_{\min }$, to the onset times, $t_{\text {onset, for the oscillations }}$ observed at ATS-1. In particular, for a given tonset we looked for the nearest $t_{\text {min }}$ and measured $\left|t_{\text {min }}{ }^{-t_{\text {onset }}}\right|$. Figure 3 illustrates the result of the study with a histogram showing the number of events versus the . time difference $\mid t_{\min }{ }^{-t}$ onset $\mid$. Half of all the events observed at ATS-1 occurred within one day of one of these local minima.

\section{THE DOMINANT MODE}

The most frequently observed period at ATS-1 is in the range 40-90 seconds, and the average period is $\mathrm{T}_{\text {ave }}=77.4$ seconds. If an oscillation with the average period corresponds to the second harmonic of a standing Alfvén wave, then according to Table 2 of Cumings et al. (1969), the average number density during the oscillations would be in the range $5.6<\mathrm{n}_{0}<15.3 / \mathrm{cm}^{3}$, depending upon how the plasma is distributed along the ATS-1 field line. Since we know that the oscillations are associated with periods of magnetic disturbance, when ATS-1 is most likely in the tough region on the dayside of the magnetosphere, these number densities are unacceptably high. Measurements of the particle density by detectors aboard ATS-5 (DeForest and McIlwain, 1971) indicate that the maximum expected density at synchronous orbit on the dayside is $n_{0} \approx 2.2 / \mathrm{cm}^{3}$, with a typical density of $n_{0} \approx 1 / \mathrm{cm}^{3}$.

If on the other hand an oscillation with the average period, $T_{\text {ave }}=77.4$, is interpreted as the fundamental mode of a standing Alfvén wave, the average 
number density would be in the range $1.4<n_{0}<2.2 / \mathrm{cm}^{3}$. These densities are much more easily reconcilled with the direct measurements made by ATS-5.

We have already noted that the oscillations observed at ATS-1 seem to occur preferentially during disturbed times. DeForest and McIlwain (1971) also point out that intrusions of hot plasma at the synchronous orbit occur during magnetic substorms. The plasma that supports the oscillations must be composed primarily of the substorm injected plasma rather than the cold plasma whose source is the ionosphere. Using OGO-5 cold plasma data, kindly supplied by Dr. C.R. Chappell, we have tried to compare a few individual cases when 0GO-5 was near ATS-1 and oscillations were recorded at ATS-1. In each case, we found that the cold plasma density was below that required to explain the oscillations even in terms of the fundamental, much less the second harmonic.

The case of April 17, 1968 is typical of those we studied. 0G0-5 was inbound on its seventeenth orbit and crossed $\mathrm{L}=6.6$ at approximately $1640 \mathrm{U} . \mathrm{T}$. near 0900 L.T. ATS-1 passed through 0900 L.T. a little more than two hours later and oscillations with an average period of 55.7 seconds were recorded. The density of cold plasma measured by 0GO-5 was approximately $n_{0}=0.4 / \mathrm{cm}^{3}$. If the oscillations observed at ATS-1 are to be interpreted as the fundamental mode of a standing Alfvén wave, then the total proton number density would have to be $\mathrm{n}_{0} \approx 1 / \mathrm{cm}^{3}$.

\section{HIGHER HARMONICS}

of the 248 events under investigation, 28 either overlapped another event or almost did so. That is, there were 14 cases when oscillations of different periods were occurring during the same time interval. These events are listed 
in Table 1, which shows the beginning time and ending time for each event, the average period, and the ratio of the larger period to the smaller period.

There are several interesting features in Table 1. First, note that most of the overlapping events occur near noon, 2200 U.T. (Recall that ATS-1 is located at approximately $150^{\circ} \mathrm{W}$ longitude. Thus, the local time is ten hours less than the universal time.) The exceptions are sets Nos. 3, 11, 12, and 14. Secondly, note that the period ratios cluster around two values, $R_{1} \approx 2.2$ and $R_{2} \approx 4.5$. The exceptions are sets Nos. 8, 12, and 14. Thirdiy, note that for all the sets that have ratios clustering around $R_{I}$ the larger period is in the range 40-90 seconds, which we identify as the dominant mode from the histogram in Figure 1.

The above observations suggest that these overlapping events represent. cases when the fundamental and a higher harmonic of the Alfvén standing waves were occurring simultaneously. It is possible to check this hypothesis bv using the simple model of Alfvén standing waves that we presented in our initial analysis (Cummings et al., 1969). Theoretical ratios of the period of the fundamental and of higher harmonics can be computed using Table 1 in that paper. These ratios, computed for the toroidal mode, are only weakly dependent on the distribution of plasma along the field lines. The theoretical ratio of the period of the fundamental to the period of the second harmonic, $\mathrm{T}_{1} / \mathrm{T}_{2}$, has a range of . 2.0 - 2.6. Six of the seven overlapping sets that cluster around $R_{1}$ fall within the above range.

The theoretical ratio of the period of the fundamental to the period of the thind harmonic, $\mathrm{T}_{1} / \mathrm{T}_{3}$, has a range of $3.0-4.3$. None of the fourteen sets of overlapping events have period ratios that fall within this range. 
The theoretical ratios of the period of the fundamental to the period of the fourth harmonic, $T_{1} / T_{4}$, has a range of $4.0-5.9$. All four of the overlapping sets that cluster around $R_{2}$ fall within this range.

Two of the three sets that do not have period ratios falling within any of the above ranges are sets Nos. 12 and 14 . Both of these sets occurred in the dusk sector, where the description of the field as a dipole is probably less valj.d than in the noon sector.

Eleven of the fourteen cases when oscillations at two different frequencies were occurring simultaneously at ATS-1 can be interpreted by our simple model as either the fundamental and the second harmonic (seven cases) or the fundamental and the fourth harmonic (four cases). As noted above, for all seven cases that we interpret as the simultaneous occurrence of the fundamental and the second harmonic, the larger period is in the range 40-90 seconds. This is the dominant range of periods observed at ATS-1; more than half of all events recorded have periods in this range. We thus have added evidence that the dominant mode observed at ATS-1 is the fundamental mode of a standing Alfvén wave.

We also note here from the histogram of Figure 1, that much of the peak in the period range $20-30$ seconds is attributable, to the occurrence of the second harmonic. In five of the seven cases of the simultaneous occurrence of the fundamental and the second harmonic the period of the higher harmonic was in this range.

\section{SUMMARY AND CONCLUSIONS}

The oscillations observed at ATS-1 most frequently occurred with periods in the range 40 90 seconds. The great majority of the events recorded at ATS-1 occurred on the dayside of the synchronous orbit. The onset of the oscillations 
seems to be associated with a disturbed magnetosphere, rather than a quiet one as previously supposed. The current best estimates of the total proton number density on the dayside of the synchronous orbit are consistent with the interpretation of the dominant mode of oscillations observed at ATS-1 as the fundamental mode of a standing Alfvén wave. From the few cases when oscillations of distinctly different periods occurred simultaneously, we conclude that the second and fourth harmonics can also occur.

\section{Acknowledgments}

The authors have benefited from helpful discussions with Drs. C.R. Chappell, R.L. McPheron, and P.J. Coleman, Jn. This research was supported in part by the National Aeronautics and Space Administration under grant NGR-10-011-007. 


\section{REFERENCES}

Barfield, J.N., R.L. McPheron, P.J. Coleman, Jr, and D.T. Southwood, StormAssociated Pc5 Micropulsations Events Observed at the Synchronous Equatorial Orbit, J. Geophys. Res., 77, 143, 1972.

Cummings, W.D., R. Mason and P.J. Coleman, Jr., Some Characteristics of LowFrequency Oscillations Observed at ATS-1, J. Geophys. Res., 77, 748, 1972.

Cummings, W.D., R.J. O'Sullivan and P.J. Coleman, Jr., Standing Alfvén Waves in the Magnetosphere, J. Geophys. Res., 74, 778, 1969.

DeForest, S.E. and C.E. McIlwain, Plasma Clouds in the Magnetosphere, J. Geophys. Res., 76, 3593, 1971.

Dwarkin, M.L., A.J. Zmuda and W.E. Radford, Hydromagnetic Waves at 6.25 Earth Radii With Periods Between 3 and 240 Seconds, J. Geophys. Res., 76, 3668, 1971.

Lanzerotti, L.J. and H. Fukuniski, Modes of Magnetospheric Alfvén Waves, Reviews of Geophysics and Space. Physics, 12, 724,1974.

Sugiura, M and D.J. Poros, Hourly Values of Equatorial Dst for the Years 1957 to 1970, Publication No. 645-71-278, Goddard Space Flight Center, July, 1971. 
Table I

Overlapping Events at ATS-1

Set Beginning Time (U.T.) Ending Time (U.T.) No. Mon. Day Hr. Min. Mon. Day Hr. Min.

1 Jan. $02 \quad 19 \quad 06 \quad$ Jan. $02 \quad 22 \quad 56$

Jan. $01 \quad 18 \quad 20$

2 Jan. $18 \quad 23 \quad 09$

Jan. $19 \quad 00 \quad 59$

3 Jan. $20 \quad 06 \quad 28$

Jan. $20 \quad 08 \quad 15$

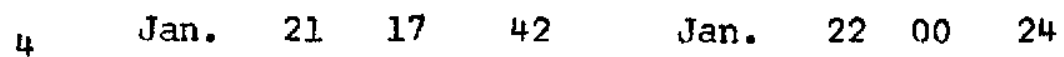

Jan. $21 \quad 18 \quad 12$

5 Jan. $22 \quad 15 \quad 22$

Jan. $22 \quad 16 \quad 09$

6 Jan. $23 \quad 21 \quad 59$

Jan. $23 \quad 17 \quad 08$

7 Mar. $01 \quad 21 \quad 10$

Mar. $01 \quad 20 \quad 22$

8 Mar. $29 \quad 19 \quad 52$

Mar. $29 \quad 18 \quad 20$

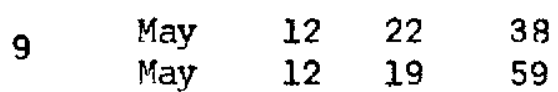

10

May $13 \quad 20 \quad 26$

May $\quad 13 \quad 19 \quad 14$

11 May $16 \quad 00 \quad 59$

May $\quad 16 \quad 00 \quad 16$

$12 \begin{array}{llll}\text { June } & 09 & 03 & 25 \\ \text { June } & 09 & 04 & 1\end{array}$

$13^{2}$ June $11 \quad 17 \quad 10$

June $1120 \quad 42$

$\begin{array}{lllll}14 & \text { June } & 17 & 00 & 0 \\ \text { June } & 17 & 00 & 26\end{array}$

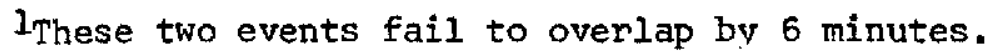

${ }^{2}$ These two events fail to overlap by 20 minutes.
Average Period

Jan. $02 \quad 23 \quad 43$

Jan. $\quad 1901 \quad 05$

Jan. $1901 \quad 12$

Jan. $20 \quad 10 \quad 02$

Jan. $20 \quad 08 \quad 23$

Jan. $2200 \quad 19$

Jan. $22 \quad 18 \quad 06$

Jan. 23 OI 05

Jan. $\quad \begin{array}{lll}24 & 01 & 12\end{array}$

Jan. 24 01 54

Mar. $\quad 02 \quad 00 \quad 10$

Mar. $\quad 01 \quad 21 \quad 24$

Mar. $\quad 29 \quad 22 \quad 55$

Mar. $\quad 2922 \quad 20$

May $\quad \begin{array}{lll}12 & 23 & 10\end{array}$

May $1300 \quad 15$

May $\quad 1401 \quad 10$

May $\begin{array}{lll}13 & 22 & 39\end{array}$

May $\quad 16 \quad 01 \quad 20$

May $\quad 16 \quad 00 \quad 53$

June $\quad 09 \quad 04 \quad 59$

June $09 \quad 04 \quad 33$

June $\quad 11 \quad 20 \quad 22$

June $11 \quad 23 \quad 23$

$\begin{array}{llll}\text { June } & 17 & 02 & 28\end{array}$

June $\begin{array}{lll}17 & 01 & 35\end{array}$
$354.3 \pm 81.3$

$80.5 \mp 12.5$

$63.9+5.4$

$28.1 \pm 1.9$

$88.4+9.3$

$44.8 \pm 7.1$

$52.6+5.9$

$24.4 \pm 2.9$

$46.2+7.4$

$24.4 \pm 2.2$

$160.6+19.3$

$31.4 \pm 6.5$

$295.2+45.2$

$61.8 \pm 3.3$

$410.8 \pm 19.3$

$53.4 \pm 6.3$

$55.5+7.3$

$26.4 \mp 4.8$

$76.8+9.1$

$30.2 \pm 4.8$

$59.7+8.7$

$26.6 \pm 0.6$

$30.5 \pm 23.8$
$79.8 \pm 6.2$

$290.0+5.9$

$64.0 \pm 13.5$

$101.3+6.0$

$82.6 \pm 2.3$
2.2

1.6

4.5

Ratio

of Period

4.4

2.3

2.0

2.2

1.9

5.1

4.6

7.7

2.1

2.5

1.2 
Figure 1 The histogram shows the number of events recorded at ATS-1 with periods occurring in each ten second interval from 20 to 250 seconds. As explained in the text, oscillations with periods below about 20 seconds are often obscured by an artificial oscillation, and are, therefore not included in this study. The data interval for Figures 1,2 , and 3 is January-June, 1968, and compressional storm-related oscillations are excluded.

Figure 2 The histogram shows the number of hours in which oscillations were recorded at ATS-1 for each hour of local time.

Figure 3 The histogram of this figure was constructed in the following way. The onset time, $t_{\text {onset, }}$ for each event was recorded and compared to the time of the nearest minimum in Dst, $t_{\mathrm{min}}$. The amplitude of the excursion in Dst at the minimum point was required to be at least $35 \gamma$. The histopram shows the number of events recorded at ATS-1 for each four-hour interval of $\left(t_{\min }-t_{\text {onset }}\right)$. A negative value of ( $t_{\min }-t_{\text {onset }}$ ) means the onset of the event was prior to the nearest minimum in bst. 


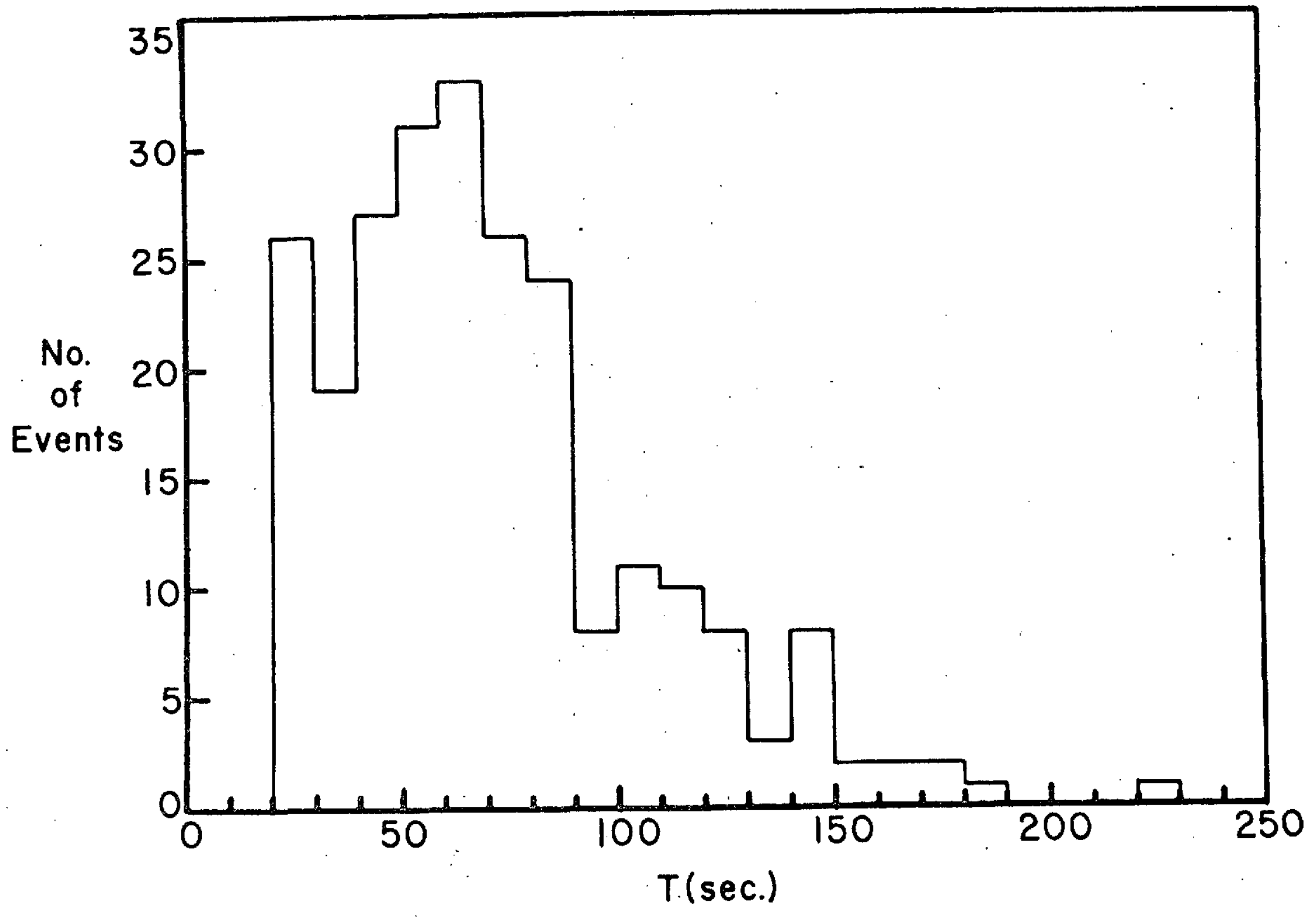

Figure 1 


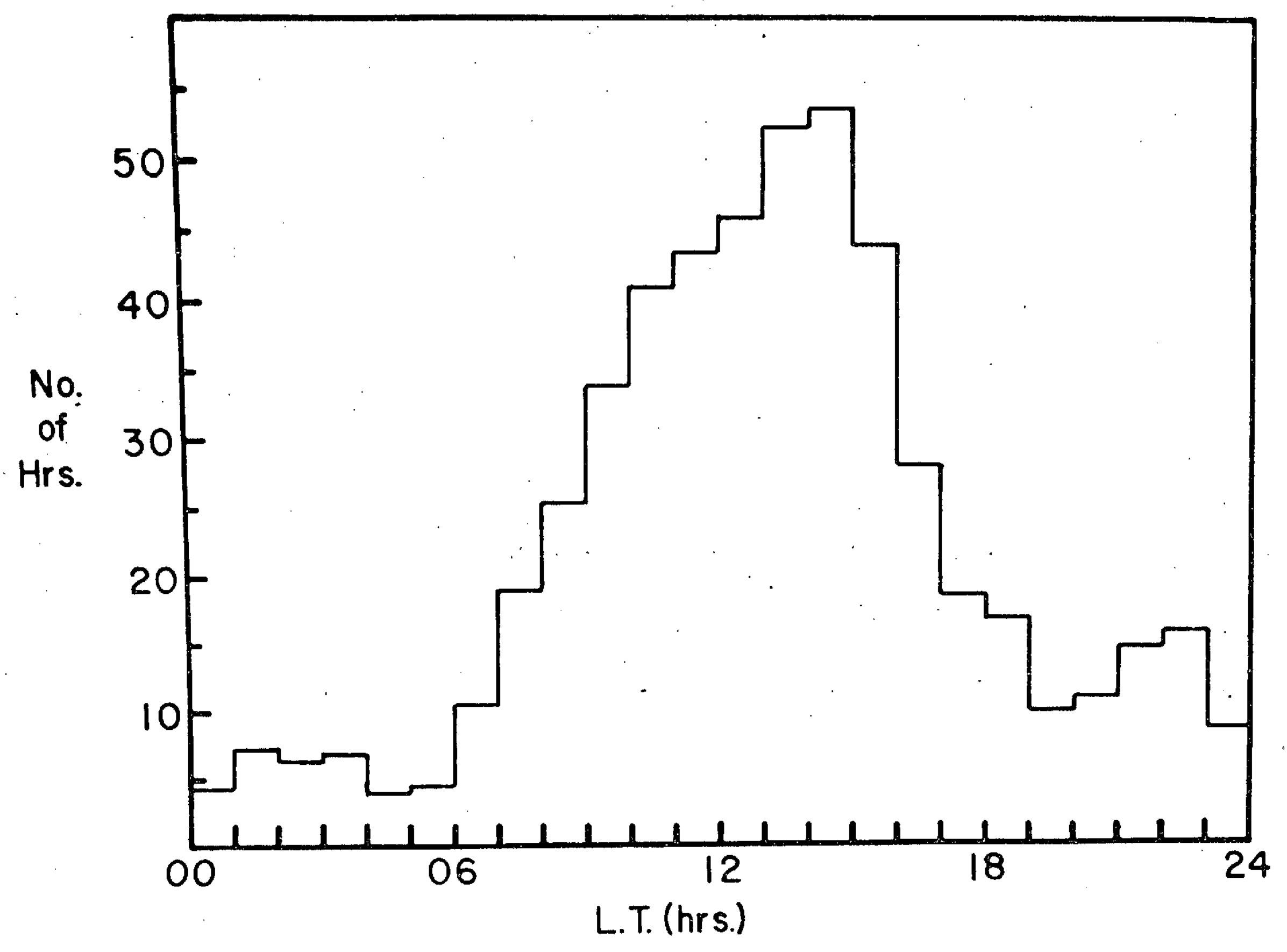




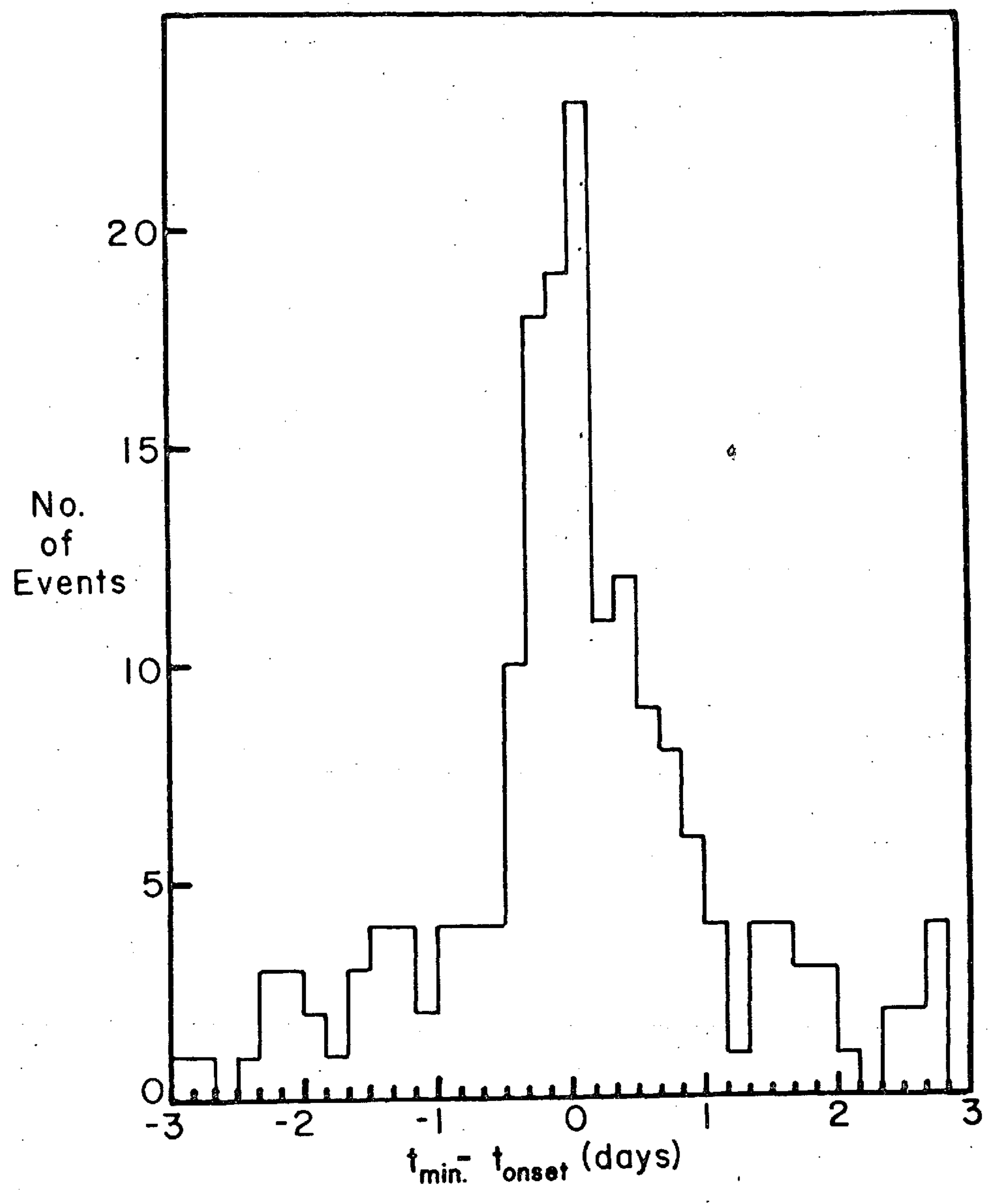

Figure 3 